\title{
EDITORIAL
}

\section{The Man - Made Healthcare Disaster}

There has been a remarkable progress in the field of medicine, particularly in recent past. But this technological advancement has come at a price. Since past few decades, health care system worldwide has seen a steady increase in costs that have burdened nations. In this scenario, tobacco use has emerged as one of the biggest public health threats the world has ever seen.

Globally, tobacco use is increasing despite gradually declining in the developed countries. The epidemic of tobacco use is now shifting to the developing world. Currently, there are more than a billion smokers in the world, with more than 80 percent smokers living in low and middle-income countries. Tobacco use kills 5.4 million people annually accounting for about 10 percent adult deaths worldwide, thus killing up to half of all users, and becoming a leading preventable cause of death globally. The tobacco-related deaths are expected to increase to more than eight million per year by 2030, with 80 of such deaths occurring in the developing nations. It has caused an estimated 100 million deaths in 20th century, and with current trends it is expected to kill up to one billion in the current century.

Smoking harms nearly every organ of the body. It causes many diseases and reduces the health of smokers in general. It is a risk factor for six of the eight leading causes of deaths in the world. It increases risk for death from all causes in men and women. As the health consequences of tobacco use appear after many years, it seems that the epidemic of disease and death has just begun. The healthcare scenario is going to worsen in coming years, more so in developing countries.

As it is the single largest preventable cause of death and diseases, there is an urgent need to wage a war against this man-made disaster. No longer, one has to accept it as a personal choice of those who smoke, as it directly and indirectly affects almost everyone in the society including small children through passive smoking. The task at hand is really huge, but not impossible if we act collectively at all levels to control it.

Dr. Chandra P. Dokwal

Sr. Consultant \& Coordinator

Department of Respiratory Medicine

Apollo Hospitals Dhaka 